\title{
Radiation risks from large solar energetic particle events
}

\author{
R. A. Mewaldt ${ }^{1}$, C. M. S. Cohen ${ }^{1}$, D. K. Haggerty ${ }^{2}$, G. M. Mason², \\ M. L. Looper ${ }^{3}$, T. T. von Rosenvinge ${ }^{4}$, and M. E. Wiedenbeck ${ }^{5}$ \\ ${ }^{I}$ California Institute of Technology, Pasadena CA 91125 \\ ${ }^{2}$ Johns Hopkins University/Applied Physics Laboratory, Laurel MD 20723 \\ ${ }^{3}$ The Aerospace Corporation, Los Angeles CA 90009 \\ ${ }^{4}$ NASA Goddard Space Flight Center, Greenbelt MD, 20771 \\ ${ }^{5}$ Jet Propulsion Laboratory, California Institute of Technology, Pasadena CA 91109
}

\begin{abstract}
Solar energetic particles (SEPs) constitute a radiation hazard to both humans and hardware in space. Over the past few years there have been significant advances in our knowledge of the composition and energy spectra of SEP events, leading to new insights into the conditions that contribute to the largest events. This paper summarizes the energy spectra and frequency of large SEP events, and discusses the interplanetary conditions that affect the intensity of the largest events.
\end{abstract}

Keywords: solar energetic particles, particle acceleration, coronal mass ejections, radiation risks PACS: $96.50 . \mathrm{Pw}, 96.50 . \mathrm{Vg}, 96.50 . \mathrm{sh}, 96.60 . \mathrm{vg}, 96.50 .96 .60 \mathrm{ph}$

\section{INTRODUCTION}

During large solar energetic particle (SEP) events the intensity of $>10 \mathrm{MeV}$ protons in nearby interplanetary space can increase by $>10^{5}$ times over that of galactic cosmic rays (see Figure 1), creating a radiation hazard to both humans and hardware in space. With NASA now committed to sending astronauts back to the Moon, outside the shelter of Earth's magnetosphere, there is renewed interest in understanding and forecasting large SEP events. The past solar maximum included four of the top ten SEP events of the space era. Fortunately, the array of spacecraft now in interplanetary space has provided greatly improved measurements of the composition and energy spectra of accelerated ions, leading to fresh insights into the nature of these events.

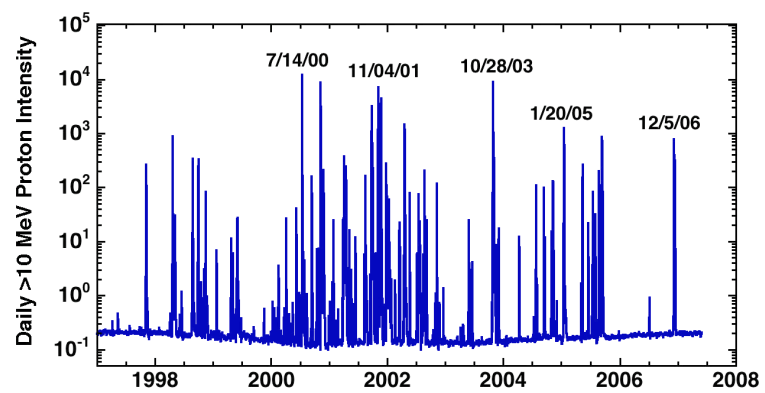

FIGURE 1: Daily proton intensities measured by GOES. Five large SEP events are labeled.

CP932, Turbulence and Nonlinear Processes in Astrophysical Plasmas

$6^{\text {th }}$ Annual International Astrophysical Conference, edited by D. Shaikh and G. P. Zank

(C) 2007 American Institute of Physics 978-0-7354-0443-4/07/\$23.00 
The largest SEP events are due to particles accelerated by coronal and interplanetary shocks driven by coronal mass ejections (CMEs) traveling at $>2000$ $\mathrm{km} / \mathrm{sec}$. Although shock acceleration is ubiquitous in nature, its efficiency is highly variable, making it difficult to forecast large SEP events. This paper will describe the energy spectra of the largest SEP events, discuss their frequency of occurrence, consider a worst-case SEP event, and discuss the interplanetary conditions that affect the intensity of these events.

\section{SOLAR ENERGETIC PARTICLE CHARACTERISTICS}

During solar cycle 23 new instrumentation made it possible for the first time to measure the composition and energy spectra of SEP events over a broad energy interval $(<0.1$ to $>100 \mathrm{MeV} /$ nuc), including all abundant elements from $\mathrm{H}$ to $\mathrm{Fe}$. Although heavy ions up through the Fe-group often present the greatest risk to electronic hardware in space, for radiation risks to humans protons make up $\sim 90 \%$ of the risk due to SEP events because they are the most abundant and because they are more difficult to shield against than heavier ions.

During the last solar maximum it became apparent that the spectra of all large solar proton events are power-laws at low energies $(<1 \mathrm{MeV})$ with typical spectral indices of -1 to -2 , followed by a break in the spectrum, typically between $\sim 3$ and $\sim 30 \mathrm{MeV}$. Figure $2 \mathrm{a}$ includes energy spectra for some of the large SEP events from solar cycle 23. Above the spectral break some events appear to have an exponential fall-off (giving an Ellison-Ramaty shape [1]), but many appear to have a second power-law that continues to several hundred $\mathrm{MeV}[2,3,4]$, such as for the December 2006 event in Figure 2a measured by ACE, STEREO, and SAMPEX [5]. Such spectra (as well as the October 28, 2003 event in Figure 2a) are better fit by the double power-law function of Band et al. [6] (see examples in [2,3,4]). Note that the spectral break in the December 2006 event is at lower energy, but there is a smaller change in slope. It is not yet understood how the high-energy power law originates.
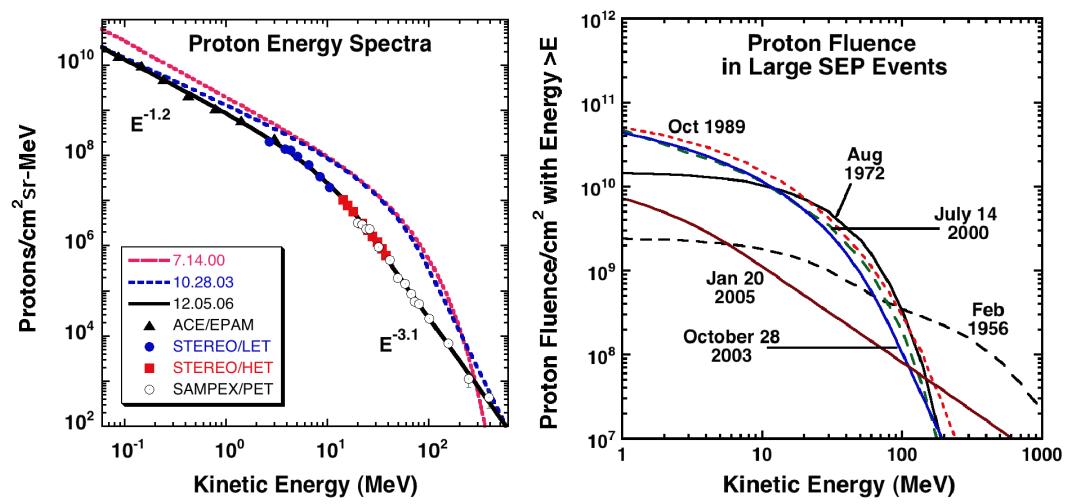

FIGURE 2: (a): Fits to proton differential energy spectra for three large SEP events, including data for the Dec. 5, 2006 event from ACE, STEREO, and SAMPEX. (b) Integral energy spectra of some of the largest SEP events of the last 50 years, including three events from solar cycle 23 (adapted from Mewaldt et al. 2006 [7]) 
In Figure $2 b$ three events from solar cycle 23 are compared to some of the largest events of the space era. For purposes of astronaut safety it is conventional to characterize SEP events by the fluence of $>30 \mathrm{MeV}$ protons, with the critical energy range being $\sim 30$ to $\sim 200 \mathrm{MeV}$ [8]. The largest SEP event of the space era is usually taken to be the August 1972 event, with a $>30 \mathrm{MeV}$ fluence of $5 \times 10^{9}$ protons $/ \mathrm{cm}^{2}$. Solar cycle 23 included four events with $>3 \times 10^{9}$ protons $/ \mathrm{cm}^{2}$, all of which are within the top 10 of the last 50 years.

Although there are only 60 years of direct measurements of SEP events (including ion chamber, neutron monitor, balloon, and spacecraft data), McCracken et al. $[9,10]$ have extended the SEP record back to $\sim 1460$ using measurements of the nitrate concentration in polar ice cores (nitrates produced by SEPs in the upper atmosphere precipitate and are incorporated in polar ice). In Figure 3a, 11-year averages of SEP fluences since 1956 are shown with 44-year averages inferred from ice-core data [10]. The space era has included somewhat below-average SEP fluences until recently.

The combined data set can be used to calculate the occurrence probability for SEP events of a given size. Figure $3 b$ shows the probability versus SEP fluence for 192-day periods, the longest lunar mission that NASA is presently considering. A possible design requirement is to ensure that astronauts have sufficient shielding to not exceed dose limits during events with an occurrence probability of $>1 \%$. With this criterion the shielding would need to be sufficient for events with $\sim 8 \times 10^{9}$ protons $/ \mathrm{cm}^{2}>30$ $\mathrm{MeV}$ if missions are to take place at solar maximum. Solar minimum missions would require less shielding.

As an example, Kim et al. [12] find that for an interplanetary spacecraft during the August 1972 event it would take $\sim 15 \mathrm{~g} / \mathrm{cm}^{2}$ of aluminum shielding to reduce the radiation dose below the present 30-day limit of 0.25 Gy (assuming spherical geometry). On the lunar surface (where the Moon provides shielding over $\sim 2 \pi \mathrm{sr}$ ), the necessary shielding would be reduced to $\sim 5-8 \mathrm{~g} / \mathrm{cm}^{2}$ of aluminum $[8,12]$.
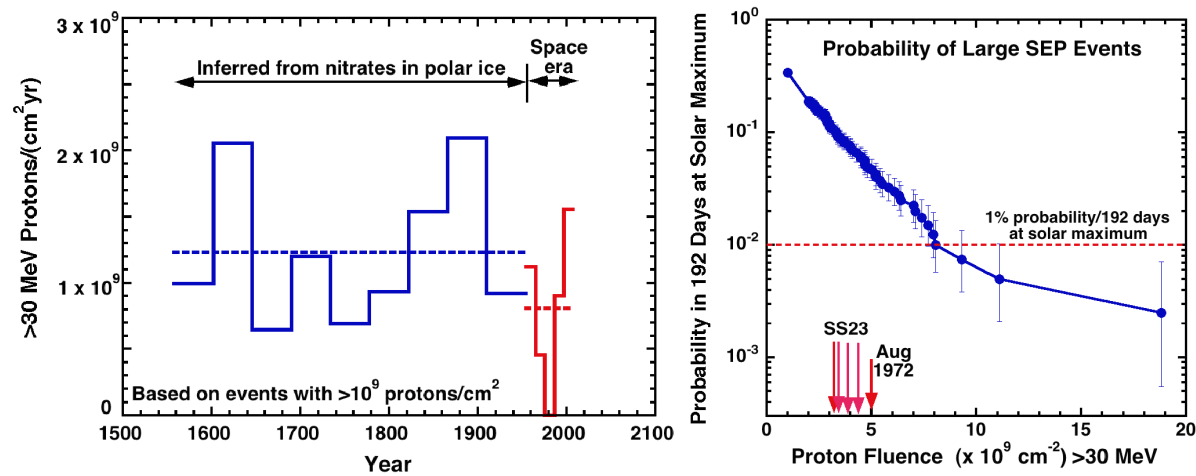

FIGURE 3: (a) The $>30 \mathrm{MeV}$ proton fluence over the past 450 years (based on events with $>30 \mathrm{MeV}$ fluences of $>10^{9}$ protons $/ \mathrm{cm}^{2}$ ). Data from 1560 to 1954 are 44 -year averages of nitrate measurements in polar ice caps $[9,10]$. The modern data are a combination of neutron monitor, polar-cap absorption, and spacecraft data (especially 1976-2007 data from NOAA's GOES satellites (updated from [11])). (b) The long-term average probability of experiencing an SEP event of a given size in 192 days at solar maximum, based on a combination of data from polar ice cores and in situ observations during the modern era. Fluences for August 1972 and four events from solar cycle 23 are indicated. 


\section{SEP STREAMING LIMITS}

One of the foundations of particle acceleration at parallel shocks is that energetic ions streaming away from the shock will generate turbulence by amplifying existing Alfven waves, thereby trapping particles near the shock and increasing the acceleration efficiency [13]. A consequence of this process is that the intensity of protons escaping upstream from the shock may be subject to "streaming limits" as a result of self-generated turbulence. Reames and $\mathrm{Ng}$ [14] used GOES data to establish empirical streaming limits for protons in several energy ranges. The January 20, 2005 SEP event served as a wake-up call when the $>100 \mathrm{MeV}$ proton intensity near Earth reached maximum within $\sim 30$ minutes of the onset of the X-ray flare (see Figure $4 a$ ), resulting in the largest ground-level neutron monitor event since February 1956 ([15], see also the energy spectra in Figure 2b). This event had a much faster rise time than other large SEP events (Figure 4a), and it also exceeded the 110-500 MeV streaming limit established by Reames and $\mathrm{Ng}$ by a factor of $\sim 4$. It is possible that streaming limits depend on the level of pre-existing turbulence, or on the proton energy spectrum (this event had a very hard spectrum (see [16] and Figure 2a)).
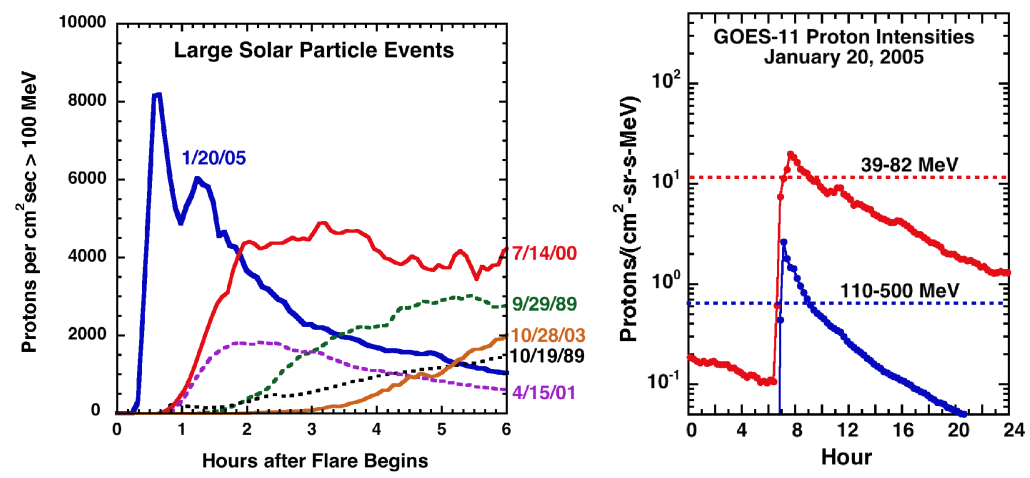

FIGURE 4: (a) The January 20, 2005 event recorded the highest $>100 \mathrm{MeV}$ intensity in the history of NOAA's GOES satellites, and reached maximum intensity much faster than any other large events [16]. (b) The 110-500 MeV proton intensity measured by GOES-11 during the January 20 , 2005 event exceeded the "streaming limit" (dotted line) of Reames and $\mathrm{Ng}$ [14] by a factor of $\sim 4$.

\section{WORST CASE SEP EVENTS}

The largest event in the polar ice cap record occurred in 1859 , coinciding with the 1859 white-light flare observed by Carrington and the largest geomagnetic storm on record. The $>30 \mathrm{MeV}$ fluence for this event was conservatively estimated to be 19 x $10^{9}$ protons $/ \mathrm{cm}^{2}$ [9], or $\sim 4$ times that of the August 1972 event (see Figure 2b). It is of interest to consider whether an event this large requires an unreasonably fast or massive CME relative to those recorded in the SOHO data base.

The energy source for accelerating SEPs is derived from the mechanical energy of the CME. Mewaldt et al. [2,17] compared the total energy in accelerated particles to the kinetic energy of the CME $[18,19]$ for 17 SEP events and found that in many large 
events $\sim 10 \%$ of the $\mathrm{CME}$ kinetic energy goes into accelerated particles (see Figure 5a). The SOHO/LASCO instrument has observed $>10,000 \mathrm{CMEs}$ since 1996. The maximum inferred values of the mass, velocity, and kinetic energy of SOHO CMES [20] are $2 \times 10^{16} \mathrm{~g}, 3387 \mathrm{~km} / \mathrm{s}$, and $\sim 10^{33}$ ergs, respectively (the estimated velocity of the January 20, $2005 \mathrm{CME}$ is $3675 \mathrm{~km} / \mathrm{s}$ [21]). In Figure $5 \mathrm{~b}$ we show the estimated SEP intensity for various combinations of CME mass and velocity, assuming $10 \%$ SEP acceleration efficiency. Estimates are done for both October 28, 2003 and January 20, 2005 spectra (see Figure 2b). Note that the Carrington event does not require a CME mass or velocity outside the LASCO data base (although the required kinetic energy would be several times greater). For example, a mass of $3 \times 10^{16} \mathrm{~g}$, and a velocity of $3000 \mathrm{~km} / \mathrm{s}$ would be sufficient with a hard spectrum. The SEP acceleration efficiency could also exceed $10 \%$. Based on these considerations, the Carrington event should probably not be considered an extreme anomaly.
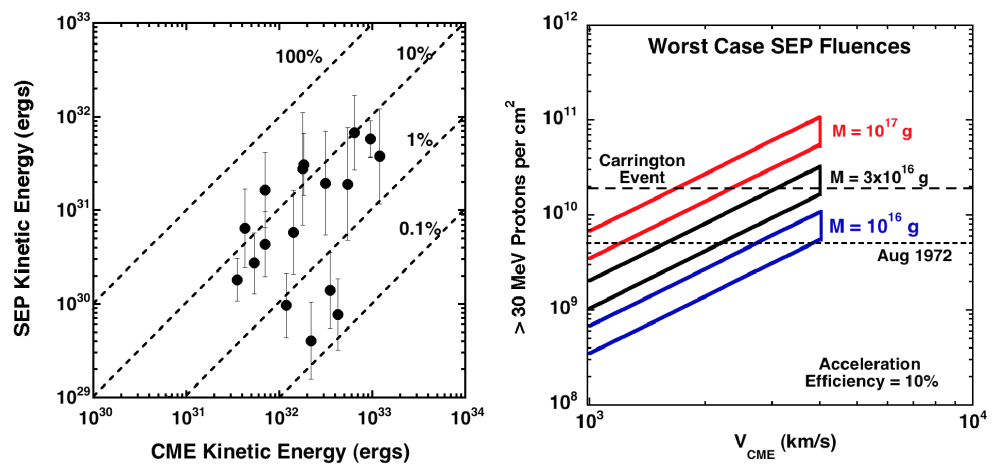

FIGURE 5: (a) Comparison of SEP and CME kinetic energies for 17 SEP events [18]. (b) Estimated $>30 \mathrm{MeV}$ proton fluence for various combinations of CME mass and velocity, assuming $10 \%$ acceleration efficiency. For a given CME mass there is a factor of $\sim 2$ variation based on whether the October 28, 2003 or January 20, 2005 spectra are assumed (Figure 2).

\section{DISCUSSION}

In addition to the factors considered above, the following conditions may affect the intensity of large SEP events.

CME-velocity: Kahler [22] finds the peak intensity of $20 \mathrm{MeV}$ protons scales with CME velocity as $\left(\mathrm{V}_{\mathrm{cme}}\right)^{4.86}$, with orders of magnitude scatter about this fit at a given CME velocity. For many fast CMEs there is no SEP event. It will be interesting to see how the correlation in Figure 5a develops when additional events are included.

Seed particle populations: It now appears that most large SEP events are accelerated from the suprathermal tail of the solar wind rather than the thermal plasma $[23,24,25]$. The density of seed particles in this region varies by several orders of magnitude [24] and (at least for Fe) there appears to be a correlation between event fluence and the density of suprathermal Fe prior to the event [26].

Pre-existing turbulence: Gopalswamy et al. [27] found that the shock acceleration efficiency is apparently much higher when there has been an earlier CME from the 
same active region during the previous day. Li and Zank [28] suggested that this may be due to enhanced interplanetary turbulence levels resulting from the first CME, an idea that should be tested with in situ turbulence data. Gopalswamy et al [27] have also suggested other possibilities.

Location: The intensity and rise time of an SEP event at Earth depend on the magnetic connection to the acceleration region [29]. In large SEP events the highest intensities often occur when the shock reaches the observer (e.g., the August 1972 and October 1989 events). Correcting for longitude, the (east-limb) December 5, 2006 event (Figure 2a) moves from $11^{\text {th }}$ to within the top 5 of solar cycle 23 .

The STEREO mission is ushering in a new era of multipoint imaging and in situ measurements that can test many of these ideas, including the longitude distribution of SEPs, the correlation between SEP and CME kinetic energies, and the effects of multiple CMEs on particle acceleration. Measurements of CMEs and SEPs from three points of view rather than one should lead to a global picture of SEP acceleration and transport and enable improved forecasting of the onset and evolution of these events.

Acknowledgements: This work was supported by NASA under NAS5-03131, NAG5-12929, NNG04GB55G and NNX06AC21G. We appreciate the use of GOES data provided by NOAA and thank the organizers for an excellent conference.

\section{REFERENCES}

1. D. C. Ellison and R. Ramaty, Ap. J., 298, 400-408 (1985).

2. R. A. Mewaldt, et al.: J. Geophys. Res., 110, doi:10.1029/2005JA011038 (2005).

3. A. J. Tylka, et al., Ap. J. Suppl., 164, 536-551 (2006).

4. R. A. Mewaldt, C. M. S. Cohen, G. M. Mason et al., in The Physics of Collisionless Shocks, AIP Conf. Proceedings \#781, G. Li et al., editors, pp. 227-232 (2005).

5. R. A. Mewaldt, et al., submitted to the 30 th Internat. Cosmic Ray Conf. (2007).

6. D. Band et al. (1993), Ap. J., 413, 281-292, 1993.

7. R. A. Mewaldt, Space Science Reviews, DOI: 10.1007/s11214-006-9091-0 (2006).

8. R. E. Turner, in Solar Eruptions and Energetic Particles, N. Gopolswamy, R. A. Mewaldt, and J. Torsti, eds, AGU Monograph Series, p. 367-374 (2006).

9. K. G. McCracken et al., J. Geophys. Res. 106, 21585-21598 (2001).

10. K. G. McCracken et al., J. Geophys. Res. 106, 21599-21610 (2001).

11. M. A. Shea and D. F. Smart, Solar Physics 127. 297-320 (1990).

12. M. Y. Kim, X. Hu, and F. A. Cucinotta: American Institute of Aeronautics and Astronatics, Space 2005 Conference, Paper AIAA-6653, pp. 1-16 (2005).

13. M. A. Lee, J. Geophys. Res. 88, 6109-6119 (1983).

14. D. V. Reames, and C. K. Ng, Ap.J. 504, 1002-1005 (1998).

15. J. W. Bieber, et al., Am. Geophys. Union, Fall Meeting 2005, Abstract \#SH21A-03.

16. R. A. Mewaldt, M. D. Looper, C. M. S. Cohen, et al. Proc. 29th Internat. Cosmic Ray Conf. 1, 111-114 (2005).

17. R. A. Mewaldt et al., Solar Wind-11/SOHO-16 Conf. (ESA SP-592), B. Fleck, et al., eds., pp 67-70 (2005).

18. N. Gopalswamy et al., J. Geophys. Res., 109, A12105, doi:10.1029/2004JA10602 (2004).

19. A. Vourlidas, P. Subramanian, K. P. Dere, and R. A. Howard, Ap. J., 534, 456 (2000).

20. S. Yashiro, SOHO LASCO CME Catalog at http://cdaw.gsfc.nasa.gov/CME list/

21. N. Gopalswamy, H. Xie, S. Yashiro, and I. Usoskin, Proc. 29th Internat. Cosmic Ray Conf. 1, 169-172 (2005).

22. Kahler, S. W., J. Geophys Res. 106, 20,947-20,955 (2001).

23. G. M. Mason, Space Science Reviews, 99, 119-133 (2001).

24. R. A. Mewaldt, C. M. S. Cohen, and G. M. Mason, in Solar Eruptions and Energetic Particles, N. Gopalswamy, R. A. Mewaldt, and J. Torsti, eds, AGU Monograph Series, pp. 115-125 (2006).

25. M. I. Desai et al., Ap. J. 649, 470-489 (2006).

26. R. A. Mewaldt et al., $36^{\text {th }}$ COSPAR Scientific Assembly, Beijing, p. 3540 (2006).

27. N. Gopalswamy et al. J. Geophys. Res. 109. doi:10.10029/2004JA010602 (2004).

28. G. Li and G. P. Zank, Proc. 29th Internat. Cosmic Ray Conf. 1, 173-176 (2005).

29. H. V. Cane and D. Lario, Space Science Reviews 123, 45-56 (2006). 